\title{
A new self-scaling VM-algorithm for non-convex optimization, part 1
}

\author{
Prof Dr. Abbas Y. AL-Bayati \\ Department of mathematics, \\ College of Computers Sciences and Mathematics \\ University of Mosul, IRAQ \\ \& \\ Dr. Maha S.Y. AL-Salih \\ Department of Computers Science / College of Education \\ University of Mosul, IRAQ
}

Received

06 / 05 / 2010
Accepted

02 / 03 / 2011

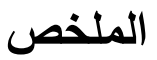

خوارزمية المتري المتغير ذاتي القياس تحل مسائل الامتلية غير الخطية وغير المقيدة

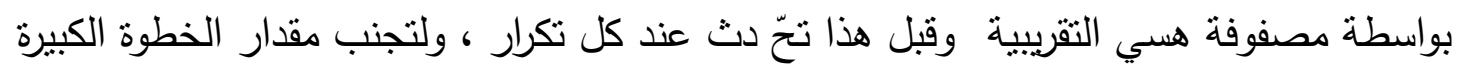
لمصفوفات هسي النقربيية لدالة الهدف ـ قمنا ببرهان هذه الخوارزميات في حالة التقارب الثشام ل والسرعة فوق الخطية عندما تكون الدالة غير محدبة.

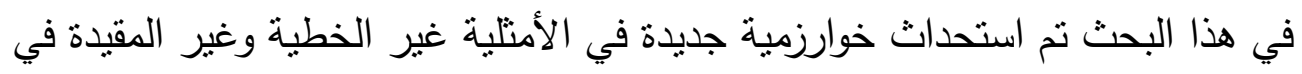

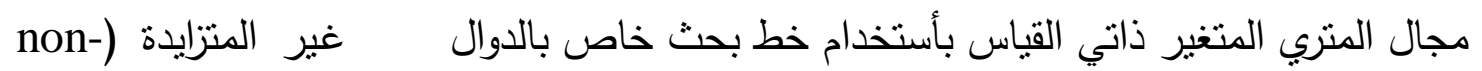
(monotone line search للخوارزمية المقترحة في الامثلية غير الدحدبة.
\end{abstract}

\begin{abstract}
The self-scaling VM-algorithms solves an unconstrained non-linear optimization problems by scaling the Hessian approximation matrix before it is updated at each iteration to avoid the possible large eigenvalues in the Hessian approximation matrices of the objective function $\mathrm{f}(\mathrm{x})$.It has been proved that these algorithms have a global and superlinear convergences when $f(x)$ is non- convex.

In this paper we are going to propose a new self-scaling VMalgorithm with a new non-monotone line search procedure with a detailed study of the global and super-linear convergence property for the new proposed algorithm in non-convex optimization.

Keywords: VM-methods, non-monotone line searches, self-scaling ALBayati VM- method, global converge, super-linear convergence.
\end{abstract}




\section{Introduction}

We study the global convergence of a self -scaling Al-Bayati [4], VM- method with non-monotone line searches for solving the unconstrained optimization problem (see[3]and[12])

$$
\min _{x \in R^{n}} f(x)
$$

where $\mathrm{f}$ is a continuously differentiable function of $\mathrm{n}$ variables. At the $\mathrm{k}^{\text {th }}$ iteration of the self-scaling method, asymmetric and positive definite matrix $B_{k}$ is given, and a search direction is computed by

$$
d_{k}=-B_{k}^{-1} g_{k}
$$

where $g_{k}$ is the gradient of $f$ evaluated at the current iterate $x_{k}$. One then computes the next iterate by

$$
x_{k+1}=x_{k}+\alpha_{k} d_{k}
$$

where the step size $\alpha_{\mathrm{k}}$ satisfies the wolfe conditions

$$
f\left(x_{k}+\alpha_{k} d_{k}\right) \leq f\left(x_{k}\right)+\delta_{1} \alpha_{k} d_{k}^{T} g_{k}
$$

and

$$
\mathrm{g}\left(\mathrm{x}_{\mathrm{k}}+\alpha_{\mathrm{k}} \mathrm{d}_{\mathrm{k}}\right)^{\mathrm{T}} \mathrm{d}_{\mathrm{k}} \geq \delta_{2} \mathrm{~d}_{\mathrm{k}}^{\mathrm{T}} \mathrm{g}_{\mathrm{k}}
$$

Where $0<\delta_{1}<\frac{1}{2}$ and $\delta_{1}<\delta_{2}<1$.

and satisfies another conditions as we explain it later.

\section{Variable Metric Method}

The theory of Variable Metric methods is beautiful. We now have a fairly good understanding of their properties. Much of this knowledge has been obtained recently, and we will discuss it in this paper.

The BFGS method is a line search method. At the k-th iteration, a symmetric and positive $B_{k}$ is given and a search direction is computed by (1.2) and (1.3). It has been found that it is best to implement BFGS formula (Broyden (1970), Fletcher (1970), Goldfarb (1970) and Shanno (1970) ), (see [1], [2] ).

Where

$$
B_{k+1}=B_{k}-\frac{B_{k} v_{k} v_{k}^{T} B_{k}}{v_{k}^{T} B_{k} v_{k}}+\frac{y_{k} y_{k}^{T}}{y_{k}^{T} v_{k}}
$$

$$
\mathrm{y}_{\mathrm{k}}=\mathrm{g}_{\mathrm{k}+1}-\mathrm{g}_{\mathrm{k}} \text { and } v_{k}=x_{k+1}-x_{k}
$$

The analysis has been extended by Byrd, et. al. (see [11]) to restricted Broyden class of Quasi-Newton method in (1.6) which is replaced by

$$
B_{k+1}=B_{k}-\frac{B_{k} v_{k} v_{k}^{T} B_{k}}{v_{k}^{T} B_{k} v_{k}}+\frac{y_{k} y_{k}^{T}}{y_{k}^{T} v_{k}}+\phi\left(v_{k}^{T} B_{k} v_{k}\right) v_{k} v_{k}^{T}
$$

where $\phi \in[0,1]$, and

$v_{k}=\left[\frac{y_{k}}{y_{k}^{T} v_{k}}-\frac{B_{k} v_{k}}{v_{k}^{T} B_{k} v_{k}}\right]$ 
The choice $\phi=0$ gives rise to the BFGS update where as $\phi=1$ defines the DFP method, the first Variable Metric method proposed by Davidon, Fletcher and Powell (see Fletcher, [13]). And then the Hessian approximation is then updated by Al-Bayati [4].

$$
B_{k+1}=B_{k}+\frac{B_{k} v_{k} v_{k}^{T} B_{k}}{v_{k}^{T} B_{k} v_{k}}+\rho_{k} \frac{y_{k} y_{k}^{T}}{v_{k}^{T} y_{k}^{T}}
$$

where

$$
\rho_{k}=\frac{v_{k}^{T} B_{k} v_{k}}{y_{k}^{T} v_{k}} \text { (for more details see Al-Bayati [4]) }
$$

Note that this is done only for a quadratic model. But for non quadratic models, see (Al-Bayati [5], Al-Bayati \& Al-Assady [6] and Al-Bayati [7]). For the constraint optimization problems and scaled sequential BFGS algorithm see (AL-Bayati and Hammed [8]).

\section{A new Non-monotones self-scaling Al-Bayati (1991) VM- algorithm}

First we give the outline of the above algorithm as follows:

\section{Algorithm (2.1)}

At the $\mathrm{k}^{\text {th }}$ iteration denote $\mathrm{f}_{\mathrm{k}}=\mathrm{f}\left(\mathrm{x}_{\mathrm{k}}\right)$ and $\mathrm{g}_{\mathrm{k}}=\mathrm{g}\left(\mathrm{x}_{\mathrm{k}}\right)$

Step (0) For given $\mathrm{x}_{\mathrm{o}}$ and initial symmetric positive definite matrix $\mathrm{H}_{\mathrm{o}}$ let $\mathrm{k}=0$

Step (1) If $\left\|g_{k}\right\|=0$ stop

Step (2) Determine the search direction

$$
\mathrm{d}_{\mathrm{k}}=-\mathrm{B}_{\mathrm{k}}^{-1} \mathrm{~g}_{\mathrm{k}}
$$

Step (3) Find step length $\alpha_{k}$ by a new line search approach (NLS) below

Step (4) Generate a new iteration point by

$$
\mathrm{x}_{\mathrm{k}+1}=\mathrm{x}_{\mathrm{k}}+\alpha_{\mathrm{k}} \mathrm{d}_{\mathrm{k}}
$$

Step (5) Update the Hessian matrix $G_{k}$ by the following Al-Bayati [4] self-scaling

VM- update

$$
\begin{gathered}
B_{k+1}=B_{k}-\frac{B_{k} v_{k} v_{k}^{T} B_{k}}{v_{k}^{T} B_{k} v_{k}}+\rho_{k} \frac{y_{k} y_{k}^{T}}{v_{k}^{T} y_{k}} \\
v_{k}=x_{k+1}-x_{k} \\
y_{k}=g_{k+1}-g_{k} \\
\rho_{k}=\frac{v_{k}^{T} B_{k} v_{k}}{v_{k}^{T} y_{k}}
\end{gathered}
$$

Step (6) Let $\mathrm{k}=\mathrm{k}+1$ and go to step (1).

Now we produce a new non-monotone line search approach to determine the step- length in step (3). For this we introduce the concept of forcing function. 
Definition 2.1: The mapping $\sigma:[0,+\infty] \rightarrow[0,+\infty]$ is called a forcing function, if for any sequence $\left\{\mathrm{t}_{\mathrm{i}}\right\}$ with $\mathrm{t}_{\mathrm{i}} \geq 0$ we have

$$
\lim _{i \rightarrow \infty} \sigma\left(t_{i}\right)=0 \Rightarrow \lim _{i \rightarrow \infty} t_{i}=0
$$

\subsection{A new non-monotone line search (NLS) approach}

let $\sigma_{1}, \sigma_{2}$ be two forcing functions

let $\mathrm{m}$ be a positive integer

Given parameters $0<\delta_{1}<\beta<1$ and $\mathrm{M} \geq 0, \delta_{2}>0$

At the iteration $\mathrm{k}$, the step length $\alpha_{\mathrm{k}}$ (for more details see[9]).

satisfies that:

$$
\begin{gathered}
\mathrm{f}\left(\mathrm{x}_{\mathrm{k}}+\alpha_{\mathrm{k}} \mathrm{d}_{\mathrm{k}}\right) \leq \mathrm{C}_{\mathrm{k}}-\delta_{1} \min \left\{\sigma_{1}\left(\mu_{\mathrm{k}}\right), \sigma_{2}\left(\zeta_{\mathrm{k}}\right)\right\}-\delta_{2} \alpha_{\mathrm{k}}^{2}\left\|\mathrm{~d}_{\mathrm{k}}\right\|^{2} \\
\mathrm{~g}\left(\mathrm{x}_{\mathrm{k}}+\alpha_{\mathrm{k}} \mathrm{d}_{\mathrm{k}}\right)^{\mathrm{T}} \mathrm{d}_{\mathrm{k}} \geq \beta_{\mathrm{k}} \mathrm{g}_{\mathrm{k}}^{\mathrm{T}} \mathrm{d}_{\mathrm{k}} \\
\mu_{\mathrm{k}}=-\frac{\mathrm{g}_{\mathrm{k}}^{\mathrm{T}} \mathrm{d}_{\mathrm{k}}}{\left\|\mathrm{d}_{\mathrm{k}}\right\|} \quad \zeta_{\mathrm{k}}=-\alpha_{\mathrm{k}} \mathrm{g}_{\mathrm{k}}^{\mathrm{T}} \mathrm{d}_{\mathrm{k}}
\end{gathered}
$$

$\mathrm{C}_{0}=\mathrm{f}_{\mathrm{o}}, \mathrm{Q}_{\mathrm{o}}=1$ and $\mathrm{C}_{\mathrm{k}+1}=\frac{\gamma_{\mathrm{k}} \mathrm{Q}_{\mathrm{k}} \mathrm{C}_{\mathrm{k}}+\mathrm{f}_{\mathrm{k}+1}}{\mathrm{Q}_{\mathrm{k}+1}}$

with $\gamma_{\mathrm{k}} \in[0,1]$ and

$$
\mathrm{Q}_{\mathrm{k}+1}=\gamma_{\mathrm{k}} \mathrm{Q}_{\mathrm{k}}+1
$$

The updating of $\mathrm{C}_{\mathrm{k}}$ in (2.11) was recently given by Zhang and Hager [15]. Clearly from (2.11) and (2.12) $C_{k}$ is a convex combination of the function values $f_{o}, f_{1}, \ldots, f_{k}$.

The choice of $\gamma_{\mathrm{k}}$ controls the degree of the non-monotonicity If $\gamma_{\mathrm{k}}=0$ and $\min \left\{\sigma_{1}, \sigma_{2}\right\}=\zeta_{\mathrm{k}}$ for each $\mathrm{k}$ then (2.8) reduces to the monotone line search. If $\gamma_{k}=1$ for each $k$ then $C_{k}$ is the average of $f_{o}, f_{1}$, $\ldots, \mathrm{f}_{\mathrm{k}}$ and (2.8) reduces to the monotone non-increasing function.

\section{Theorem 2.4:}

Consider $x_{k+1}=x_{k}+\alpha_{k} d_{k}, \alpha_{k}$ satisfying (2.8)-(2.10) if $\left\{f_{k}\right\}$ in infinite and bounded below then

$$
\sum_{k=0}^{\infty} \frac{\min \left\{\sigma_{1}\left(\mu_{k}\right), \sigma_{2}(\zeta)\right\}+\alpha^{2}\left\|d_{k}\right\|^{2}}{Q_{k+1}}<\infty
$$

Proof:

From (2.8) it is clear that $\alpha_{\mathrm{k}}^{2}\left\|\mathrm{~d}_{\mathrm{k}}\right\|^{2} \geq 0$ and since $\mathrm{d}$ is descent then $\mu_{\mathrm{k}} \geq 0$ and $\zeta_{\mathrm{k}} \geq 0$

$$
\begin{gathered}
\Rightarrow \min \left\{\sigma_{1}, \sigma_{2}\right\} \geq 0 \\
\Rightarrow \mathrm{f}_{\mathrm{k}+1} \leq \mathrm{C}_{\mathrm{k}} \text { from }(2.8)
\end{gathered}
$$

and 


$$
\begin{array}{r}
\mathrm{C}_{\mathrm{k}+1}=\frac{\gamma_{\mathrm{k}} \mathrm{Q}_{\mathrm{k}} \mathrm{C}_{\mathrm{k}}+\mathrm{f}_{\mathrm{k}+1}}{\mathrm{Q}_{\mathrm{k}+1}} \\
\leq \frac{\gamma_{k} Q_{k} C_{k}+\left\{C_{k}-\min \left\{\sigma_{1}, \sigma_{2}\right\}-\alpha_{k}^{2}\left\|d_{k}\right\|^{2}\right\}}{Q_{k+1}} \\
\leq \frac{C_{k}\left\{\gamma_{k} Q_{k}+1\right\}}{Q_{k+1}}-\frac{\min \left\{\sigma_{1}, \sigma_{2}\right\}+\alpha_{k}^{2}\left\|d_{k}\right\|^{2}}{Q_{k+1}}
\end{array}
$$

from (2.12) we get

$\leq C_{k}-\frac{\min \left\{\sigma_{1}, \sigma_{2}\right\}+\alpha_{k}^{2}\left\|d_{k}\right\|^{2}}{Q_{k+1}}$

$\therefore\left\{\mathrm{C}_{\mathrm{k}}\right\}$ is monotone non-increasing since $\left\{\mathrm{f}_{\mathrm{k}}\right\}$ is bounded from (2.15) $\left\{\mathrm{f}_{\mathrm{k}}\right\}$ is non- increasing (see [10]). This implies

$$
\sum \frac{\min \left\{\sigma_{1}, \sigma_{2}\right\}+\alpha_{k}^{2}\left\|d_{k}\right\|^{2}}{Q_{k+1}}<\infty
$$

\section{The global convergence property of the new proposed algorithm (2.1)}

Let us consider the following

\section{Assumption 3.1}

(1) for a given $x_{1} \in R^{n}$

let the level set $S=\left\{x \in R^{n}: f(x) \leq f\left(x_{1}\right)\right\}$ be a bounded

(2) In some neighborhood $\mathrm{N}(\mathrm{S})$ of $\mathrm{S}$. $\mathrm{g}(\mathrm{x})$ satisfies Lipschitz continuous condition, i.e.

$$
\begin{gathered}
\text { for all } x, \bar{x} \in N(S) \\
\|g(x)-g(\bar{x})\| \leq L\|x-\bar{x}\|
\end{gathered}
$$

\section{Lemma 3.2}

Under assumption 3.1 if $\alpha_{\mathrm{k}}$ satisfies

$$
\mathrm{g}_{\mathrm{k}+1}^{\mathrm{T}} \mathrm{d}_{\mathrm{k}} \geq \beta \mathrm{g}_{\mathrm{k}}^{\mathrm{T}} \mathrm{d}_{\mathrm{k}} \quad 0<\beta<1
$$

then $\alpha_{k} \geq \frac{1-\beta}{L} \frac{\mu_{k}}{\left\|d_{k}\right\|}$.

\section{Proof:}

Clearly

$\left(g_{k+1}-g_{k}\right)^{T} d_{k} \leq\left\|g_{k+1}-g_{k}\right\|\left\|d_{k}\right\|$

from (3.2) $\leq L\|x-\bar{x}\|\left\|d_{k}\right\|$

$$
\left\|\mathrm{d}_{\mathrm{k}}\right\|^{2} \leq \mathrm{L} \alpha_{\mathrm{k}}
$$

Also from (3.3) we can write 
$\mathrm{g}_{\mathrm{k}+1}^{\mathrm{T}} \mathrm{d}_{\mathrm{k}}-\mathrm{g}_{\mathrm{k}}^{\mathrm{T}} \mathrm{d}_{\mathrm{k}} \geq \beta \mathrm{g}_{\mathrm{k}}^{\mathrm{T}} \mathrm{d}_{\mathrm{k}}-\mathrm{g}_{\mathrm{k}}^{\mathrm{T}} \mathrm{d}_{\mathrm{k}}$.

$\Rightarrow\left(\mathrm{g}_{\mathrm{k}+1}-\mathrm{g}_{\mathrm{k}}\right)^{\mathrm{T}} \mathrm{d}_{\mathrm{k}} \geq(\beta-1) \mathrm{g}_{\mathrm{k}}^{\mathrm{T}} \mathrm{d}_{\mathrm{k}} \geq 0$

since $0<\beta<1$

from (3.5) and (3.6) we have

$$
\begin{gathered}
\mathrm{L} \alpha_{\mathrm{k}}\left\|\mathrm{d}_{\mathrm{k}}\right\|^{2} \geq(\beta-1) \mathrm{g}_{\mathrm{k}}^{\mathrm{T}} \mathrm{d}_{\mathrm{k}} \\
\Rightarrow \alpha_{\mathrm{k}} \geq \frac{\beta-1 \mathrm{~g}_{\mathrm{k}}^{\mathrm{T}} \mathrm{d}_{\mathrm{k}}}{\mathrm{L}\left\|\mathrm{d}_{\mathrm{k}}\right\|^{2}}
\end{gathered}
$$

from (2.10) we have

$$
\alpha_{k} \geq \frac{1-\beta}{L} \frac{\mu_{k}}{\left\|d_{k}\right\|}
$$

Now before starting the proof of the global convergence of the proposed algorithm, we define an auxiliary matrix sequence $\left\{\bar{B}_{k}\right\}$ as Nocedal and Yuan did in [14], assume that $\overline{\mathrm{B}_{1}}=\mathrm{B}_{1}$ and

$$
\overline{\mathrm{B}}_{\mathrm{k}+1}=\frac{\mathrm{v}_{\mathrm{k}}^{\mathrm{T}} \mathrm{y}_{\mathrm{k}}}{\mathrm{v}_{\mathrm{k}}^{\mathrm{T}} \overline{\mathrm{B}} \mathrm{v}_{\mathrm{k}}} \mathrm{B}_{\mathrm{k}+1}, \mathrm{k} \geq 1
$$

from (2.1) we have

$\mathrm{v}_{\mathrm{k}}=-\overline{\alpha_{\mathrm{k}}} \overline{\mathrm{B}_{\mathrm{k}}} \mathrm{g}_{\mathrm{k}}$

where

$$
\overline{\alpha_{\mathrm{k}}}=\frac{\mathrm{v}_{\mathrm{k}-1}^{\mathrm{T}} \mathrm{y}_{\mathrm{k}-1}}{\mathrm{v}_{\mathrm{k}-1}^{\mathrm{T}} \overline{\mathrm{B}}_{\mathrm{k}-1}^{-1} \mathrm{v}_{\mathrm{k}-1}} \alpha_{\mathrm{k}}
$$

since $\rho_{\mathrm{k}}>0$ then if $v_{k}^{T} y_{k}>0 \Rightarrow \bar{B}_{k+1}$ is a positive definite

Lemma 3.3 : From [12]

For $\left\{\bar{B}_{k}\right\}$ defined in (3.10) we have

$$
\begin{gathered}
\operatorname{tr}\left(\bar{B}_{k+1}\right)=\operatorname{tr}\left(\bar{B}_{k}\right)-\frac{\left\|\bar{B}_{k} v_{k}\right\|^{2}}{v_{k}^{T} \bar{B}_{k} v_{k}}+\frac{y_{k}^{T} v_{k}}{v_{k}^{T} \bar{B}_{k} v_{k}}+\frac{\mathrm{y}_{\mathrm{k}}^{\mathrm{T}} \mathrm{v}_{\mathrm{k}}}{\mathrm{v}_{\mathrm{k}}^{\mathrm{T}} \overline{\mathrm{B}}_{\mathrm{k}} \mathrm{v}_{\mathrm{k}}} \frac{\left\|\mathrm{y}_{\mathrm{k}}\right\|^{2}}{\mathrm{y}_{\mathrm{k}}^{\mathrm{T}} \mathrm{v}_{\mathrm{k}}} \\
\overline{\mathrm{B}}_{\mathrm{k}+1}=\overline{\mathrm{B}}_{\mathrm{k}}-\frac{\overline{\mathrm{B}}_{\mathrm{k}} \mathrm{v}_{\mathrm{k}} \mathrm{v}_{\mathrm{k}}^{\mathrm{T}} \overline{\mathrm{B}}_{\mathrm{k}}}{\mathrm{v}_{\mathrm{k}}^{\mathrm{T}} \overline{\mathrm{B}}_{\mathrm{k}} \mathrm{v}_{\mathrm{k}}}+\frac{\mathrm{y}_{\mathrm{k}}^{\mathrm{T}} \mathrm{v}_{\mathrm{k}}}{\mathrm{v}_{\mathrm{k}}^{\mathrm{T}} \overline{\mathrm{B}}_{\mathrm{k}} \mathrm{v}_{\mathrm{k}}} \cdot \frac{\mathrm{y}_{\mathrm{k}} \mathrm{y}_{\mathrm{k}}^{\mathrm{T}}}{\mathrm{v}_{\mathrm{k}}^{\mathrm{T}} \mathrm{y}}
\end{gathered}
$$

Then for all $\mathrm{k} \geq 1$

$$
\begin{aligned}
& \operatorname{tr}\left(\overline{\mathrm{B}}_{\mathrm{k}+1}\right)=\operatorname{tr}\left(\overline{\mathrm{B}}_{\mathrm{k}}\right)-\frac{\left\|\overline{\mathrm{B}}_{\mathrm{k}} \mathrm{v}_{\mathrm{k}}\right\|^{2}}{\mathrm{v}_{\mathrm{k}}^{\mathrm{T}} \overline{\mathrm{B}}_{\mathrm{k}} \mathrm{v}_{\mathrm{k}}}+\frac{\mathrm{y}_{\mathrm{k}}^{\mathrm{T}} \mathrm{v}_{\mathrm{k}}}{\mathrm{v}_{\mathrm{k}}^{\mathrm{T}} \overline{\mathrm{B}}_{\mathrm{k}} \mathrm{v}_{\mathrm{k}}}\left(1+\frac{\left\|\mathrm{y}_{\mathrm{k}}\right\|^{2}}{\mathrm{y}_{\mathrm{k}}^{\mathrm{T}} \mathrm{v}_{\mathrm{k}}}\right)
\end{aligned}
$$




\section{Theorem 3.3}

Let $\mathrm{x}_{1}$ be a starting point for which assumption 3.1 holds, let $\left\{\mathrm{x}_{\mathrm{k}}\right\}$ be the sequence generated by the new proposed algorithm 2.1. If there exists a positive number $\mathrm{k} \geq 1$ for which

$$
\left\|\mathrm{y}_{\mathrm{k}}\right\| \leq(1-\beta)\left\|\mathrm{g}_{\mathrm{k}}\right\|
$$

for all $\mathrm{k} \geq \mathrm{K}$ then

$$
\lim _{k \rightarrow \infty} \inf \left\|g_{k}\right\|=0
$$

\section{Proof:}

By Contradiction

Assume that there exists a positive $c_{1}>0$ for which

$$
\left\|\mathrm{g}_{\mathrm{k}}\right\| \geq \mathrm{c}_{1}
$$

For all $\mathrm{k} \geq 1$. Now since

$$
\begin{aligned}
\mathrm{v}_{\mathrm{k}}=-\bar{\alpha}_{\mathrm{k}} \overline{\mathrm{B}}_{\mathrm{k}}^{-1} \mathrm{~g}_{\mathrm{k}} \\
\Rightarrow \bar{B}_{k} v_{k}=-\bar{\alpha}_{k} g_{k}
\end{aligned}
$$

Let us start with

$\mathrm{v}_{\mathrm{k}}^{\mathrm{T}} \overline{\mathrm{B}}_{\mathrm{k}} \mathrm{v}_{\mathrm{k}}=\left(-\bar{\alpha}_{\mathrm{k}} \overline{\mathrm{B}}_{\mathrm{k}}^{-1} \mathrm{~g}_{\mathrm{k}}\right)^{\mathrm{T}}\left(-\bar{\alpha}_{\mathrm{k}} \mathrm{g}_{\mathrm{k}}\right)$

from (3.11), (3.17) and (3.18) we get

$$
=\left(-\bar{\alpha}_{k} g_{k}\right)^{\mathrm{T}}\left(-\frac{\mathrm{v}_{\mathrm{k}-1}^{\mathrm{T}} \mathrm{y}_{\mathrm{k}-1}}{\mathrm{v}_{\mathrm{k}-1}^{\mathrm{T}} \overline{\mathrm{B}}_{\mathrm{k}-1}^{-1} \mathrm{v}_{\mathrm{k}-1}} \alpha_{\mathrm{k}} \overline{\mathrm{B}}_{\mathrm{k}}^{-1} \mathrm{~g}_{\mathrm{k}}\right)
$$

From (3.10) take $\mathrm{k}+1=\mathrm{k}$ and then (2.1) we get.

$$
\Rightarrow v_{k}^{T} \bar{B}_{k} v_{k}=-\bar{\alpha}_{k} \alpha_{k} g_{k}^{T} d_{k}
$$

Now from (3.6) we have

$$
\begin{aligned}
& \mathrm{y}_{\mathrm{k}}^{\mathrm{T}} \mathrm{d}_{\mathrm{k}} \geq(\beta-1) \mathrm{g}_{\mathrm{k}}^{\mathrm{T}} \mathrm{d}_{\mathrm{k}} \\
& \Rightarrow y_{k}^{T} v_{k} \geq(\beta-1) \alpha_{k} g_{k}^{T} d_{k}
\end{aligned}
$$

substitute (3.18), (3.20) and (3.21) into (3.13)

$$
\begin{aligned}
& \operatorname{tr}\left(\bar{B}_{k+1}\right) \leq \operatorname{tr}\left(\bar{B}_{k}\right)-\frac{\left(-\bar{\alpha}_{k} g_{k}\right)^{2}}{-\bar{\alpha}_{k} \alpha_{k} g_{k}^{T} d_{k}}+\frac{(\beta-1) \alpha_{k} g_{k}^{T} d_{k}}{-\bar{\alpha}_{k} \alpha_{k} g_{k}^{T} d_{k}}+\frac{(\beta-1) \alpha_{k} g_{k}^{T} d_{k}}{-\bar{\alpha}_{k} \alpha_{k} g_{k}^{T} d_{k}} \frac{\left\|y_{k}\right\|^{2}}{(\beta-1) \alpha_{k} g_{k}^{T} d_{k}} \\
& \operatorname{tr}\left(\bar{B}_{k+1}\right) \leq \operatorname{tr}\left(\bar{B}_{k}\right)+\frac{\bar{\alpha}_{k}^{2} g_{k}^{2}}{\bar{\alpha}_{k} \alpha_{k} g_{k}^{T} d_{k}}+\frac{(\beta-1) g_{k}^{T} d_{k}}{\bar{\alpha}_{k} g_{k}^{T} d_{k}}+\frac{\left\|y_{k}\right\|^{2}}{-\bar{\alpha}_{k} \alpha_{k} g_{k}^{T} d_{k}}
\end{aligned}
$$

from (3.14) we have

$$
\begin{gathered}
\operatorname{tr}\left(\bar{B}_{k+1}\right) \leq \operatorname{tr}\left(B_{k}\right)+\frac{\bar{\alpha}_{k}\left\|g_{k}\right\|^{2}}{\alpha_{k} g_{k}^{T} d_{k}}+\frac{(1-\beta) g_{k}^{T} d_{k}}{\bar{\alpha}_{k} g_{k}^{T} d_{k}}+\frac{(1-\beta)^{2}\left\|g_{k}\right\|^{2}}{\bar{\alpha}_{k} \alpha_{k} g_{k}^{T} d_{k}} \\
\operatorname{tr}\left(\bar{B}_{k+1}\right) \leq \operatorname{tr}\left(\bar{B}_{k}\right)+\frac{\bar{\alpha}_{k}\left\|g_{k}\right\|^{2}}{\alpha_{k} g_{k}^{T} d_{k}}+\frac{(1-\beta)}{\bar{\alpha}_{k}}\left[1-\frac{(1-\beta)\left\|g_{k}\right\|^{2}}{\alpha_{k} g_{k}^{T} d_{k}}\right]
\end{gathered}
$$


since $\beta<1$ and $\mathrm{d}_{\mathrm{k}}^{\mathrm{T}} \mathrm{g}_{\mathrm{k}}<0$ clearly

$\operatorname{tr}\left(\overline{\mathrm{B}}_{\mathrm{k}+1}\right) \leq \operatorname{tr}\left(\overline{\mathrm{B}}_{\mathrm{k}}\right)$ for all $\mathrm{k} \geq \mathrm{K}$

Thus $\exists$ a constant $C_{2}>0$ for which

$$
\operatorname{tr}\left(\overline{\mathrm{B}}_{\mathrm{k}}\right) \leq \mathrm{C}_{2} \quad \text { for all } \mathrm{k}
$$

let $0<\lambda_{1}<\lambda_{2}<\ldots<\lambda_{\mathrm{n}}$ be the $\mathrm{n}$ eigen values of $\overline{\mathrm{B}}_{\mathrm{k}}$ then by (3.23)

$$
\lambda_{\mathrm{n}}\left(\overline{\mathrm{B}}_{\mathrm{k}}\right) \leq \mathrm{C}_{2}
$$

$C_{3}=\prod_{i=1}^{n} \lambda_{i}\left(\bar{B}_{k}\right) \leq \lambda_{1}\left(\bar{B}_{k}\right) C_{2}^{n-1}$

$\therefore \exists$ a constant $\mathrm{C}_{4}>0$ э

$$
\lambda_{1}\left(\bar{B}_{k}\right) \geq C_{4}
$$

i.e. $\lambda_{1}\left(\bar{B}_{k}\right)$ is the largest eigen value of the matrix $\left(\bar{B}_{k}\right)$

Now

$$
\text { Let } \overline{\mathrm{d}}_{\mathrm{k}}=-\overline{\mathrm{B}}_{\mathrm{k}}^{-1} \mathrm{~g}_{\mathrm{k}}
$$

take the norm

$$
\begin{aligned}
\left\|\overline{\mathrm{d}}_{\mathrm{k}}\right\| & \leq \frac{1}{\lambda_{1} \overline{\mathrm{B}}_{\mathrm{k}}}\left\|\mathrm{g}_{\mathrm{k}}\right\| \quad \text { by }(3.25) \\
& \leq \frac{1}{c_{4}}\left\|g_{k}\right\|,
\end{aligned}
$$

Now

$$
\begin{aligned}
-\bar{g}_{k}^{T} d_{k} & =g_{k}^{T} \bar{B}_{k}^{-1} g_{k} \\
& \geq \frac{1}{\lambda_{\mathrm{n}}\left(\overline{\mathrm{B}}_{\mathrm{k}}\right)}\left\|\mathrm{g}_{\mathrm{k}}\right\|^{2} \\
& \geq \mathrm{C}_{2}\left\|\mathrm{~g}_{\mathrm{k}}\right\|^{2}
\end{aligned}
$$

From the def. of $\mu_{k}$ and (2.10) we have

$$
\mu_{k}=\frac{-g_{k}^{T} d_{k}}{\left\|d_{k}\right\|}=\frac{-g_{k}^{T} \bar{d}_{k}}{\left\|\bar{d}_{k}\right\|} \geq \frac{C_{4}}{C_{2}}\left\|g_{k}\right\| \geq \frac{C_{1} C_{4}}{C_{2}} \geq 0
$$

from (3.16)

Also

$$
\begin{aligned}
\zeta_{\mathrm{k}} & =-\alpha_{\mathrm{k}} \mathrm{g}_{\mathrm{k}}^{\mathrm{T}} \mathrm{d}_{\mathrm{k}} \text { from (3.9) } \\
& \geq \frac{-g_{k}^{T} d_{k}}{\left\|d_{k}\right\|}\left(\frac{1-\beta}{L} \mu_{k}\right)
\end{aligned}
$$

from (2.10)

$$
\begin{gathered}
=\left(\frac{1-\beta}{\mathrm{L}}\right) \mu_{\mathrm{k}}^{2} \\
\geq\left(\frac{1-\beta}{L}\right)\left(\frac{C_{1}^{2} C_{4}^{2}}{C_{2}^{2}}\right) \succ 0
\end{gathered}
$$

Holds for any $\mathrm{k}$ 


$$
\begin{aligned}
& \text { since } \beta<1 \quad \mathrm{~L}>0 \Rightarrow \zeta_{\mathrm{k}}>0 \quad \forall_{\mathrm{k}} \\
& \Rightarrow \min \left\{\sigma_{1}\left(\mu_{\mathrm{k}}\right), \sigma_{2}\left(\zeta_{\mathrm{k}}\right)\right\}>\mathrm{C}_{5}>1 \quad \forall_{\mathrm{k}}
\end{aligned}
$$

From $\mathrm{Q}_{\mathrm{k}+1}=1+\gamma_{\mathrm{k}} \mathrm{Q}_{\mathrm{k}}$ from (2.12)

$=1+\sum_{i=0}^{k} \prod_{m=0}^{i} \gamma_{k-m} \leq k+2$

$\rightarrow \sum \frac{\min \left(\sigma_{1}, \sigma_{2}\right)+\alpha_{k}^{2}\left\|d_{k}\right\|^{2}}{Q_{k+1}} \geq \sum_{k=0}^{\infty} \frac{C_{5}}{k+2}>\infty$

which contradicts (2.17)

so we complete the proof

Remark: we can say that the above theorem will be true for the following case: depending on determinant of the matrix $B_{k}$ since

$$
\begin{aligned}
& \operatorname{det}\left(B_{k+1}^{A l-\text { Bayati }}\right)=\rho_{k} \operatorname{det}\left(B_{k}^{B F G S}\right) \\
& \text { with } \rho_{k}=\min \left\{\frac{y_{k}^{T} B_{k} y_{k}}{y_{k}^{T} v_{k}}, 1\right\}
\end{aligned}
$$

similarly, be taking the auxiliary matrix $\overline{\mathrm{B}}_{\mathrm{k}}$ as

$$
\bar{B}_{1}=B_{1}, \bar{B}_{k+1}=\max \left\{\frac{y_{k}^{T} v_{k}}{v_{k}^{T} \bar{B}_{k}^{T} v_{k}}, 1\right\} B_{k+1} ; \quad \mathrm{k} \geq 1
$$

we claim that (3.38) and (3.39) are superior on the actual BFGS update.

\section{References}

1) Al-Baali, M., (1998): Global and super-linear convergence of a restricted class of self-scaling methods with in exact line searches for convex functions. Comput. Optim. Appl.,Vol.9, pp.191-203.

2) Al-Baali, M., (1998): Numerical experience with a class of selfscaling quasi- Newton algorithms. JOTA, Vol.96, pp.533-553.

3) Al-Baali, M., (2001): On the behavior of a combined extraupdating/self-scaling BFGS method. J. Comput. Appl. Math., Vol.134, pp.269-281.

4) Al-Bayati, A. Y., (1991): A new family of self-scaling variable metric algorithms for unconstrained optimization, Journal of Educ. and Sci., Iraq, Vol.12, pp.25-54. 
5) Al-Bayati, A. Y., (1993): Anew non-quadratic model for unconstrained nonlinear optimization, J. of Mu'tah, Jordan, Vol.8, pp.131-155.

6) Al-Bayati, A. Y. and Al-Assady, (1994): Minimization of extended quadratic functions with inexact line searches, JOTA, Vol.82, pp.139-147.

7) Al-Bayati, A. Y., (2001): New generalized CG-methods for the non-quadratic model in unconstrained optimization, J. of AlYarmouk, Jordan, Vol.10, pp.1-17.

8) Al-Bayati, A. Y. and Hamed, E. T., (1998): New self-scaling sequential algorithms for the minimization of constrained non linear functions, J.of Dirasat, Jordan, Vol.25, pp.339-351.

9) Al-Bayati, A. Y. and Al-Salih, M. S., (2007): Global and superlinear convergent VM-algorithms for nonlinear optimization, J of Kirkuk University-Scientific Studies, Vol.2, pp.88-106.

10) Birgin, E. G., Martinez, J. M. Raydan, M., (2000): Non-monotone spectral projected gradient methods on convex set. SIAM J.Optim., Vol.10, pp.1196-1211.

11) Byrd, R.; Nocedal, J. and Yuan, Y., (1987): Global convergence of a class of quasi-Newton methods on convex problems, SIAM J. Numerical analysis,Vol.24, pp.1171-1190.

12) Dai, Y. H., (2002): On the non-monotone line search. JOTA, Vol.112, pp.123-160.

13) Fletcher, R., (1987): Practical methods of optimization, John Wiley and sons, New York, Toronto and Singapore.

14) Nocedal, J., Yuan, Y., (1993): Analysis of a self-scaling quasiNewton method. Math. Program, Vol.61, pp.19-37.

15) Zhang, H., Hager, W. W., (2004):A non-monotone line search technique and its application to unconstrained optimization. SIAM J. Optim., Vol.14, pp.1043-1056.

16) Yin \& Du., (2007): The Global Convergence Of Self-Scaling BFGS Algorithm with Nonmontone Line Search for Unconstrained Non convex Optimization problems. ACTA Mathematica Sinica English Series pp 1232- 1240. 